\title{
Subsistenz, Viabilität und Sozialstaat \\ Grundzüge einer Subsistenzethik
}

\section{SEBASTIAN THIEME ${ }^{* *}$}

Der philosophischen Bedeutung folgend, beschreibt „Subsistenz“ die Fähigkeit, aus sich selbst heraus zu existieren. Der vorliegende Beitrag greift dies auf und spezifiziert, was üblicherweise als „Subsistenzrecht“ bezeichnet wird. Letzteres wird als Moralprinzip vorgestellt, das sich als Kriterium der Zumutbarkeit der Integrativen Wirtschaftsethik von Peter Ulrich verstehen lässt. Darüber hinaus werden einzelne Konsequenzen des Subsistenzrechts vor dem Hintergrund der Hartz-IV-Debatte diskutiert.

Schlagwörter: Subsistenzethik, Integrative Wirtschaftsethik, Viabilität, Zumutbarkeit, Sozialtransfers

\section{Subsistence, Viability and Welfare}

According to the philosophical tradition of the term, "subsistence" describes the ability of the existence by itself. The article concentrates on this term and specifies what is commonly called "right to subsistence". The latter will be introduced as a moral principle which might be interpreted as a specification of the criterion of "reasonableness" (Zumutbarkeit) within Peter Ulrich's Integrative Economics Ethics. In addition, the article discusses some consequences of the right to subsistence with respect to the German debate about social benefits (Hartz IV).

Keywords: Subsistence Ethics, Integrative Economics Ethics, Viability, Reasonability, Social Benefits

\section{Einleitung}

Spätestens seit der Einführung des vierten Teils der sogenannten Hartz-Gesetze (2005) war die bundesdeutsche Debatte um den Sozialstaat in steter Regelmäßigkeit mit der Frage konfrontiert, was dem Menschen zum Leben zustehen soll. Für gewöhnlich taucht in diesen Diskussionen auch die Forderung nach einem Mindestlohn auf, die mit dem Argument, die Menschen müssen von ihrer eigenen Hände Arbeit leben können, den gleichen Problemkreis ansteuert. Ähnlich verhält es sich in Situationen, in denen Politikerinnen und Politiker mit Sparprogrammen einen Staatshaushalt sanieren wollen, da sich die Frage stellt, wie weit die Kürzungen beim Sozialstaat gehen dürfen. Brisant wird diese Frage vor allem in wirtschaftlichen Krisenzeiten, in denen viele Menschen von Erwerbslosigkeit bedroht sind und sie gerade deshalb den

\footnotetext{
* Beitrag eingereicht am 17.06.2013; nach doppelt verdecktem Gutachterverfahren überarbeitete Fassung angenommen am 24.01.2014.

** Dr. Sebastian Thieme, Zentrum für Ökonomische und Soziologische Studien (ZÖSS), Universität Hamburg, Von-Melle-Park 9, D-20146 Hamburg, E-Mail: sebastian.thieme@wiso.unihamburg.de, Forschungsschwerpunkte: Subsistenz(ethik), ökonomische Misanthropie, wissenschaftstheoretische und ideengeschichtliche Aspekte der Ökonomik, Soziale Marktwirtschaft.
} 
stützenden Arm eines Sozialstaats benötigen. ${ }^{1}$ Dementsprechend besitzt die Frage nach der menschlichen Selbsterhaltung einen immer wieder aktuellen Charakter. Der vorliegende Beitrag möchte daran anknüpfen, diesen Problemkomplex aber vor dem Hintergrund des allgemeinen Moralprinzips eines Subsistenærechts diskutieren.

In diesem Kontext werden die nachfolgenden Überlegungen versuchen, in kompakter Form eine Subsistenzethik zu skizzieren, die sich an der Integrativen Wirtschaftsethik von Peter Ulrich $(2000 ; 2008)$ orientiert. Diese Subsistenzethik zielt darauf ab, die Zumutbarkeit - wie sie als ein Kriterium der ethischen Legitimierbarkeit von zentraler Bedeutung ist - auszubauen. ${ }^{2}$ Zunächst werden dazu die notwendigen Begrifflichkeiten und deren Verbindung zur Integrativen Wirtschaftsethik erörtert (Abschnitt 2). Es folgt ein Exkurs zur ökonomischen Rechtfertigung des Subsistenzrechts (Abschnitt 3). Nachfolgend wird sukzessive eine Subsistenzethik entwickelt, die zusammen mit dem Subsistenzrecht als (überpositives) Moralprinzip vorzustellen ist (Abschnitt 4). Eine Diskussion über Sozialtransfers soll skizzieren, welche sozialpolitischen Implikationen sich aus einer Subsistenzethik ableiten (Abschnitt 5). Der Beitrag endet mit einem Hinweis auf die Bedeutung der Subsistenz in der Ökonomik und für die Akzeptanz ökonomischer Empfehlungen.

\section{Subsistenz, Viabilität, relative Armut und Zumutbarkeit}

Der Begriff „Subsistenz“ kann auf eine lange Geschichte zurückblicken, die von Aristoteles und der Trinitätslehre von Marius Victorinus über Boëthius, Gilbert von Poitiers und Thomas von Aquin bis hin zum Grundrecht des Subsistenzrechts bei Henry Shue (1996) reicht. Die Gemeinsamkeit, die sich über diese ideengeschichtlichen Debatten hinweg abzeichnet, besteht darin, dass der Begriff ein eigenständiges Für-sichsein beschrieb (vgl. Thieme 2010). Obwohl der Begriff heute selten reflektiert und mit unterschiedlichen Inhalten aufgeladen wird, bezieht er sich im Kern immer noch auf ein eigenständiges Für-sich-sein. ${ }^{3}$ Dies stimmt mit der Definition der „Subsistenz“, wie sie sich heute in den einschlägigen Wörterbüchern und Lexika finden lässt, überein: Dort beschreibt „Subsistenz“ das Bestehen aus sich selbst heraus. Gleichzeitig gilt sie jedoch auch als veraltete Bezeichnung für „Lebensunterhalt“.

Nachfolgend soll der philosophischen Bedeutung im Sinne des Bestehens aus sich selbst heraus gefolgt werden. Dazu muss gerade im sozialstaatlichen Kontext beachtet wer-

1 So ist z.B. auch die Internationale Arbeitsorganisation (ILO) zu verstehen, als sie in einem Bericht, erschienen im April 2013, darauf hinwies, dass ,[d]urch die Verschlechterung der Lage auf den Arbeitsmärkten (...) das Risiko sozialer Unruhen“" wächst (ILO 2013: 3ff.), und sie dementsprechend u.a. den „Ausbau der passiven und aktiven Arbeitsmarktpolitik in der Eurozone“ (ILO 2013: 6) forderte.

2 Die hier dargestellte Subsistenzethik greift viele Gedanken auf, die bereits in der Dissertationsschrift des Autors beschrieben wurden. Der vorliegende Text möchte jedoch bewusst auf (allzu) häufige (Selbst-)Zitate und Verweise verzichten. Für Interessierte soll daher der Hinweis genügen, die hier angesprochenen Sachverhalte ausführlich in Thieme (2012) nachlesen zu können.

3 Die unterschiedlichen Merkmale, die der Subsistenz beigemengt werden, lassen sich ausführlich nachlesen bei Thieme (2010: 10-20). Der Charakter eines eigenständigen Für-sich-seins schlägt sich dort hauptsächlich in der Ausrichtung auf den Eigenbedarf, die Selbstversorgung und die Gewährleistung des Lebensunterhaltes nieder. 
den, diese Selbsterhaltung nicht auf die eben erwähnte zweite Bedeutung - den Lebensunterhalt, die materielle Lebensgrundlage oder das Existenzminimum - zu reduzieren. Denn bliebe die menschliche Existenz auf ihr Minimum bzw. auf die materiellen Lebensgrundlagen beschränkt, wäre allenfalls das gewährleistet, was umgangssprachlich mit „sich gerade so über Wasser halten“ bezeichnet wird. Das menschliche Individuum ist dann zwar am Leben, aber faktisch handelt es sich nur um die Aufrechterhaltung des bisherigen Lebenszustands. Diese Fähigkeit zur Gewährleistung der Selbststeuerung zur Bewahrung der eigenen Reaktionsfähigkeit und persönlichen Stabilisierung wird nachfolgend als Viabilität bezeichnet.

Die Subsistenz im Sinne des Bestehens aus sich selbst heraus umfasst aber neben der Viabilität auch die Möglichkeit, sich selbst verändern zu können, was im Falle einer sich verändernden Umwelt als notwendige Anpassungsfähigkeit interpretiert werden darf. Während die Viabilität also darauf beschränkt bleibt, den aktuellen Lebenszustand (Status Quo) aufrechtzuerhalten, zielt die Subsistenz darauf ab, langfristig lebensfähig zu bleiben, d.h. sich selbst helfen und verändern zu können. ${ }^{4}$ Die Reizreagibilität bzw. Reaktionsfähigkeit (im biologischen Sinne) des Individuums sollte daher nicht mit der Fähigkeit verwechselt werden, sich darüber hinaus auch neue Anpassungsmöglichkeiten selbst schaffen zu können - also das Spektrum der möglichen Reaktionen (d.h. die Subsistenzmöglichkeiten) zu erweitern.

Diese begriffliche Unterscheidung mag auf den ersten Blick künstlich wirken. Im Lichte der Debatten über (Mindest-)Löhne oder Sozialtransfers gewinnt sie jedoch an Kontur. Denn es stellt einen erheblichen Unterschied dar, ob ein Lohn oder ein sozialstaatlicher Regelsatz allein die Viabilität gewährleistet oder, ob er die Betroffenen tatsächlich in die Lage versetzen soll, sich und damit ihre Situation selbst verändern zu können. Dazu ist ferner zu beachten, dass wir als Menschen immer in einen sozialen Kontext hineingeboren sind und damit die Selbsterhaltung bereits nicht auf die Erhaltung der rein physischen Existenz beschränkt sein kann: Die menschliche Selbsterhaltung bezieht sich immer auf eine Existenz in einer Gesellschaft. ${ }^{5}$

Deshalb kann sich die Subsistenzperspektive nicht allein auf diejenigen Subsistenzmittel konzentrieren, die direkt der (physischen) Selbsterhaltung dienen - z.B. Nahrungsmittel, Kleidung, Obdach, aber auch Geld. Die Subsistenz ist vom institutionellen Umfeld abhängig - den Sitten, Traditionen, Rechten und Gesetzen. Darüber hinaus muss beachtet werden, dass immaterielle Dinge wie beispielsweise Reputation und Vertrauen ebenso (neue) Subsistenzstrategien eröffnen können, wie sie bei Verlust das Subsistieren einzuschränken vermögen.

4 Diese Unterscheidung orientiert sich an James C. Scott (1976: 16ff.), der eine ,subsistence crisis zone“ erwähnte, die sich dadurch definiert, dass die Betroffenen dort nicht mehr sparen können. Sie leben dann von ihrer Substanz, was impliziert, dass sie mit Betreten dieser Zone nicht automatisch an Mangelernährung leiden und direkt vom Tode bedroht sind, aber eine Verschlechterung ihrer Sicherheit, des familiären Zusammenhalts, der Subsistenzmöglichkeiten usw. erleiden und allmählich auf das ,minimum disaster level“ zusteuern. Letzteres definierte Scott (1976: 16) als ,a food supply close enough to the physiological minimum that further reductions will lead to malnutrition and early death".

5 Siehe dazu u.a. Habermas (1958), aber ebenso den Hinweis von Chalmers (2007: 128). Mit dem konkreten Bezug zur Subsistenzproblematik siehe Scott (1976: 9, 166). 
Diese weiter gefasste Perspektive auf die menschlichen Lebensnotwendigkeiten schlägt sich üblicherweise im Begriff der „relativen Armut“ nieder, der sich in Europa durchgesetzt hat (vgl. Hauser 2008: 96ff.). Relative Armut bedeutet, dass „der Lebensstandard und die Lebensbedingungen von Menschen zu weit nach unten vom durchschnittlichen Lebensstandard und den durchschnittlichen Lebensbedingungen abweichen" (Hauser 2008: 96). Die Menschen leben dann - paradoxerweise - unterhalb ihres soziokulturellen Existenzminimums. ${ }^{6}$ In diesem Falle droht ihnen die soziale Ausgrenzung, was mit der sozialstaatlichen Gewährleistung eines soziokulturellen Existenzminimums verhindert werden soll. In Deutschland umfasst dieses neben Mitteln zur Aufrechterhaltung der physischen Existenz auch Mittel zur sozialen Teilhabe. Allerdings nur soweit dies ,zur Aufrechterhaltung eines menschenwürdigen Daseins unbedingt erforderlich" (BVerfG 2010) ist. Darin zeigt sich einerseits die weiter oben erwähnte Einsicht, dass sich das Subsistieren nicht auf die physische Existenz reduzieren lässt, andererseits wäre allerdings auch zu fragen, ob ein soziokulturelles Existenzminimum allein die Viabilität in einer Gesellschaft gewährleisten oder ob es auch das Subsistieren bewerkstelligen soll. ${ }^{7}$

Die Debatten um den Sozialstaat entzünden sich dann für gewöhnlich genau an dem Punkt, an dem es darum geht, das soziokulturelle Existenzminimum zu konkretisieren bzw. ein Subsistenzmaß zu definieren. Das Problem dabei ist, dass dem Definitionsvorhaben naturgemäß bereits die Interpretationen und Wertüberzeugungen anhaften, die die (vermeintliche) Objektivität eines solchen Maßes einschränken. ${ }^{8}$ Hinzu kommt, dass sich individuelle Umstände (Alter, Schwangerschaft, körperliche oder geistige Benachteiligungen usw.) ebenfalls der Verallgemeinerung durch ein Subsistenzmaß in den Weg stellen können. Der Hinweis auf diese Schwierigkeiten sollte jedoch nicht missverstanden werden: Er zielt lediglich darauf ab, dass solche Maße nicht in Stein gemeißelt sein können, d.h. einer kritischen Überprüfung bedürfen, und eine entsprechende Kontroverse nicht zu vermeiden ist, sie stattdessen sogar eine Notwendigkeit für die ethische Legitimation eines Subsistenzmaßes darstellt.

Jedoch ist ein weiterer Hinweis erforderlich: Die Definition eines Subsistenzmaßes impliziert in aller Regel einen individuellen Anspruch auf Subsistenzmittel, der wiederum mit einer Solidaritätspflicht seitens der restlichen Gesellschaft einhergeht. ${ }^{9}$ Die Diskussion der Subsistenzproblematik scheint sich dann für gewöhnlich auf diese An-

6

Für diese Formulierung siehe Hauser (2008: 96). Die Paradoxie besteht darin, dass in einem Existenzminimum dem Begriff nach unmissverständlich zum Ausdruck kommt, dass unterhalb dieses Minimums keine Existenz möglich ist.

In diesem Falle käme es auch auf die Qualität der Teilhabechancen an: Ist es jenen, die auf einem soziokulturellen Existenzminimum leben, tatsächlich möglich, ihre Situation zu verändern?

Dieses Problem lässt sich prominent am Urteil des Bundesverfassungsgerichts vom Februar 2010 illustrieren: Im Kern ging es um die Berechnung des soziokulturellen Existenzminimums, die das Bundesverfassungsgericht für mangelhaft hielt (vgl. BVerfG 2010). Siehe in diesem Zusammenhang auch Hauser (2008: 95) zum Begriff „Armut“.

Siehe dazu die „negative“ und „positive Gerechtigkeit“ bei Ulrich Thielemann (2010: 111ff.) sowie die Unterscheidung der negativen Freiheit (Freiheit von Einschränkungen) von der positiven Freiheit, wie sie von Chalmers (2007: 127) erwähnt und bei Berlin (1995: 201-215) ausführlich beschrieben wird. 
sprüche und deren pekuniäre Höhe zu konzentrieren. Dem gegenüber kann die Subsistenzproblematik aber auch von einer anderen Seite her betrachtet werden. Es ließe sich nämlich danach fragen, was die Subsistenz einschränkt oder gar bedroht.

Dabei muss auch Beachtung finden, dass ,[j]edes Individuum (...) in eine Gesellschaft hineingeboren [wird], die bereits in dem Sinne vor ihm existiert, als sie Charakteristika besitzt, die das Individuum nicht frei wählt" (Chalmers 2007: 128). ${ }^{10}$ Daraus folgt, dass das menschliche Subsistieren von Geburt an mit Einschränkungen konfrontiert ist. Entsprechend illusionär wäre die Vorstellung unbegrenzten Subsistierens. Die Frage nach den Einschränkungen der Subsistenz wird deshalb immer eine Frage nach den zumutbaren Einschränkungen sein. Im Begriff der „Zumutbarkeit“ bietet sich damit die Möglichkeit, die Subsistenzproblematik mit der Integrativen Wirtschaftsethik von Peter Ulrich $(2000$; 2008) zu verknüpfen, da die Zumutbarkeit dort eine wesentliche Grundlage für die ethische Legitimierbarkeit von Handlungsabsichten darstellt. Diese ethische Legitimierbarkeit ist nicht mit ethischer Legitimität zu verwechseln. Legitimierbarkeit bedeutet nur, dass eine Handlungsabsicht innerhalb eines Perspektivwechsels (z.B. im Sinne des „role reversal“ von Adam Smith oder des „Kategorischen Imperativs") als zumutbar empfunden wird und damit einem offenen Diskurs zugänglich ist. ${ }^{11}$ Als ethisch legitim gilt eine Handlungsabsicht erst dann, wenn in einem Diskurs über sie Einvernehmen erzielt wurde. ${ }^{12}$

Im Kontext der Zumutbarkeit weist die Subsistenzproblematik darauf hin, dass die Handlungsabsichten innerhalb einer Gesellschaft sehr wahrscheinlich damit einhergehen können, die Subsistenzmöglichkeiten Dritter einzuschränken. Deshalb erfordert eine solche Absicht eine Reflexion im Sinne eines Perspektivwechsels und einen offenen Diskurs hinsichtlich der Fragen, von welchen Einschränkungen Dritte betroffen sind und ob diese ihnen zugemutet werden können. Als nicht zumutbar werden dann sicher jene Handlungsabsichten gelten, die Dritte soweit einschränken, dass ihnen noch nicht einmal die Viabilitätserhaltung möglich ist. Die Viabilität bildet deshalb eine Art Untergrenze der Zumutbarkeit, an der sich entscheidet, ob eine Handlungs-

10 Siehe ergänzend Scott (1976: 166), der u.a. auf die moralischen Werte, Sozialbeziehungen und auf die Erwartungen über das Verhalten anderer hinwies, mit denen der Mensch in einer Gesellschaft - bei Scott waren das landwirtschaftlich geprägte Gesellschaftsformen - konfrontiert ist.

11 Für Details zum integrativ-wirtschaftsethischen Verständnis siehe Ulrich (2008: 81-99).

12 Laut Peter Ulrich (2008: 84) ist dieser Diskurs nicht als ein „Konsensprinzip“ - im Sinne eines gesellschaftlichen Konsensprinzips - zu überschätzen (Stichwort: „konkretistisches Missverständnis“). Stattdessen liege der Sinn der Diskursethik darin, die Verständigungsprozesse „für argumentative Kritik" offenzuhalten (vgl. Ulrich 2008: 88). Kritische Leserinnen und Leser werden diese Erwiderung sicher wenig befriedigend empfinden, da Ulrichs Hinweis auf die Funktion der Offenhaltung des Verständigungsprozesses keine Antwort auf die vorgebrachten Argumente der Kritikerinnen und Kritiker bietet: Die Idee des Diskurses ziele ihnen zufolge auf ein Ideal ab, das sich in der erfahrbaren Wirklichkeit nur schwer umsetzen ließe - sowohl hinsichtlich der Diskurssituation (Einbindung aller Parteien usw.) als auch hinsichtlich des Einvernehmens. Diese Kritikerinnen und Kritiker übersehen jedoch, dass sich nicht in allen ethischen Fragen eine „korrekte“ oder eindeutige Antwort finden lässt, weshalb solche auch in einem Diskurs nur schwer zu einem Konsens führen (Stichwort: unentscheidbare Fragen). Das trifft aber nicht auf alle ethischen Fragen zu. In Ergänzung dazu siehe die nachfolgenden Überlegungen zum Bereich der Duldung ethisch nicht legitimierter Handlungen. 
absicht im integrativ-wirtschaftsethischen Sinne (noch) als zumutbar gelten kann oder den Bereich der Unzumutbarkeit betritt.

Trotz der erwähnten Schwierigkeiten bei der Bestimmung eines soziokulturellen Existenzminimums bietet eben dieses einen ersten konkreten Anhaltspunkt dafür: Wenn eine Handlungsabsicht bei Dritten dazu führt, dass ihr Existenzminimum unterlaufen wird bzw. sie dieses nicht mehr realisieren können, dann ist diese Absicht den betroffenen Personen nicht zuzumuten. Wird die Viabilität Dritter durch die Einschränkungen einer Handlungsabsicht nicht in Frage gestellt, dann besteht zumindest die Möglichkeit, dass die Einschränkungen der Subsistenz als zumutbar empfunden werden. Doch um das festzustellen, bedarf es aus integrativ-wirtschaftsethischer Sicht eines Diskurses. ${ }^{13}$ Es dürfte allerdings ebenso klar sein, dass in dem Maße, in dem die Subsistenz Dritter auf die Erhaltung der Viabilität reduziert wird, die entsprechenden Handlungsabsichten Gefahr laufen, im offenen Diskurs als unzumutbar und damit als ethisch nicht legitim eingestuft zu werden.

Darüber hinaus existiert aber noch ein weiterer Anknüpfungspunkt an die Integrative Wirtschaftsethik: Das ,gute Leben“, das das Wirtschaften zum Ziel haben soll (vgl. Ulrich 2000: 563ff.; 2008: 229). Denn gleichwohl dieses gute Leben nicht mit der Viabilität gleichzusetzen ist, so stellt die Viabilität doch die Voraussetzung dafür dar, überhaupt von einem „Leben“ sprechen zu können: Das gute Leben setzt voraus, dass sich die Menschen viabel halten. Darüber hinaus erfordert das gute Leben u.a. eine Kultivierung des Genug-Haben-Könnens (vgl. Ulrich 2008: 229) bzw. eine Selbstbegrenzung, was sich auf die eben erwähnten Überlegungen zur Zumutbarkeit der eigenen Handlungsabsichten übertragen lässt. Diese Überlegungen laufen letztlich darauf hinaus, nicht alle Handlungsabsichten auch tatsächlich umsetzen zu müssen und über den damit verbundenen ethischen Legitimitätsvorbehalt eine Kultur des Genug-HabenKönnens zu praktizieren. Insofern fokussiert die Subsistenzperspektive nicht nur die Einschränkungen, die das Subsistenzstreben Dritter betreffen, sondern es fordert die jeweiligen Akteure auch dazu auf, darüber nachzudenken, wie weit sie selbst das eigene Subsistenzstreben einschränken würden: Müssen alle Subsistenzmöglichkeiten ergriffen werden? Auf welche Subsistenzstrategien würden sie (freiwillig) verzichten?

\section{Exkurs: Ökonomische Rechtfertigungen eines Subsistenzrechts}

Obwohl die Integrative Wirtschaftsethik, an die sich die hier präsentierte Subsistenzperspektive anlehnen soll, das Primat der Ethik vertritt (vgl. Trautnitz 2008: 144; Ulrich 2000: 558), sei für einen kurzen Augenblick der ökonomischen Perspektive gefolgt, mit der die Diskussion um Subsistenzprobleme häufig konfrontiert ist. Im Gegensatz zur Integrativen Wirtschaftsethik steht diese Sichtweise zwar unter dem Primat der Ökonomik, hält aber ebenfalls Gründe für ein Subsistenzrecht bereit. Was spricht also aus ökonomischer Sicht für ein Subsistenzrecht?

Zunächst ist dazu der logische Umstand zu benennen, dass der Wirtschaft ohne solch ein Moralprinzip die Träger ökonomischer Kalküle wegsterben würden. Soll Wirtnommen, dass sie bereits während eines Perspektivwechsels als unzumutbar erkannt und entsprechend zurückgezogen würden. 
schaft kein singuläres One-Shot-Game sein, müssen sich die Akteure mindestens viabel halten, andernfalls endet das Spiel. In der Folgeperiode würde sonst keine Person existieren, die Produkte herstellen, nachfragen oder Arbeitskraft zur Verfügung stellen kann. Wird darüber hinaus davon ausgegangen, dass sich die wirtschaftlichen Akteure selbständig an Veränderungen - z.B. Konsumpräferenzen und Ressourcenknappheit anpassen können sollen, ist unmittelbar einsichtig, dass diese dazu mehr Subsistenzmittel benötigen als zu ihrer Viabilitätserhaltung notwendig wäre. Diese elementare Fähigkeit zur Selbsterhaltung geht mit der Selbststeuerung einher, die der Idee der Märkte bisweilen unterstellt wird. Die individuelle Subsistenz bildet den Träger der marktwirtschaftlichen Selbstorganisation.

Daraus folgt, dass jegliche Wirtschaftsform die Subsistenz der Gesellschaftsmitglieder zu garantieren hat. Ist eine Wirtschaftsform nicht in der Lage, dies zu gewährleisten, dann kann sie im ökonomischen Sinne auch nicht effizient sein. Die Subsistenz stellt damit eine Voraussetzung für die ökonomische Effizienz dar. Aus einer strikt wettbewerbsorientierten Sicht wird auf diese Weise die arbeitsteilige Marktwirtschaft (wieder) in den Wettbewerb gestellt, d.h. marktwirtschaftliche Lösungen stehen nun in Konkurrenz zu anderen wirtschaftlichen Organisationsformen, wie z.B. der genossenschaftlichen Produktion oder der Eigenproduktion, die sich alle am Kriterium der Subsistenz messen lassen müssen. Das stellt sicher, dass der Markt tatsächlich ökonomisch effizient sein kann: Nur diejenigen Tätigkeiten werden marktwirtschaftlich organisiert, die in der Lage sind, sich zu reproduzieren. Das Subsistenzrecht besitzt damit eine marktwirtschaftliche Hygienefunktion.

Aus institutionsökonomischer Sicht lässt sich ferner argumentieren, dass die Menschen in eine Gesellschaft hineingeboren werden, die ihnen das Subsistieren auf vielfältige Art und Weise einschränkt, und ihnen deshalb eine Kompensation dieser Einschränkungen zusteht, soll die jeweilige Gesellschaftsform als ethisch legitim angesehen und nicht in Frage gestellt werden. Diese Einschränkungen finden sich z.B. in der Akzeptanz von Eigentums- und Vermögensverhältnissen, mithin in der Rechtschaffenheit (der Befolgung von Gesetzen) oder der Teilnahme an der Arbeitsteilung, was sich u.a. auch auf die Risiken einer Humankapitalinvestition erstreckt (Berufsausbildung etc.). Das gilt umso mehr, da z.B. Umweltschutzgesetze oder Eigentumstitel verwehren können, bestimmte Ressourcen - zum Zwecke der eigenen Subsistenzerhaltung - zu nutzen (z.B. Naturressourcen oder Wohnraum). Hinzu kommt, dass im Falle einer generellen Missachtung der Rechts- und Eigentumsordnung letztlich auch die gesamtwirtschaftlichen Transaktionskosten steigen: Wirtschaften wird damit insgesamt teurer. Außerdem können bestimmte gesellschaftliche Gruppierungen ein spezielles (Eigen-)Interesse daran besitzen, dass die mit der Wirtschaftsordnung einhergehende Einkommens- und Vermögensverteilung nicht in Frage gestellt wird. Aus allen diesen Gründen lässt sich die Institution eines Subsistenzrechts - hier verstanden im Sinne eines tatsächlich kodifizierten Rechts - als Preis für die Akzeptanz der bestehenden gesellschaftlichen Verhältnisse interpretieren. Mit einem institutionalisierten Subsistenzrecht wird also der „soziale Friede“ erkauft. Das ist keineswegs eine neue Erkenntnis, sondern findet sich bereits in den Ideen des Staatsvertrages nach Thomas Hobbes und John Locke formuliert, wie sie auch heute noch in der Institutionsökonomik referenziert werden: Aus dem Subsistenzrecht leitet sich dort ein Anspruch auf 
Widerstand $\mathrm{ab}$, der innerhalb gesellschaftlich-ökonomischer Arrangements für die elementare Selbstbindungskraft der daran Beteiligten sorgt.

Diese eben genannten Gründe messen das Subsistenzrecht hauptsächlich am ökonomischen Nutzen und folgen damit dem Primat der Ökonomik. Deshalb sei nochmals ausdrücklich darauf hingewiesen, dass die hier im Beitrag vertretene Idee eines Subsistenzrechts - trotz vereinzelter Überschneidungen (z.B. mit der Argumentation der Institutionenökonomik) - das Primat der Ethik vertritt: Das Moralprinzip des Subsistenzrechts rechtfertigt sich aus ethischen Erwägungen und stellt die ökonomischen Handlungsabsichten damit unter einen ethischen Legitimationsvorbehalt.

\section{Subsistenzethik: Elemente und Rechtfertigung eines Subsistenzrechts als überpositives Moralprinzip}

Die Art der Rechtfertigung des Subsistenzrechts als überpositives Moralprinzip hängt davon ab, welche ethische Konzeption zugrunde gelegt werden soll. Der hier vorgelagerte Exkurs zur ökonomischen Rechtfertigung des Subsistenzrechts hatte deshalb zum Ziel, aufzuzeigen, welche Gründe innerhalb einer Ethik nach ökonomischer Methode (Homann) für ein Subsistenzrecht anzuführen sind. Dagegen könnte ein Subsistenzrecht z.B. mit explizitem Hinweis auf die Bedürfnisnatur des Menschen auch naturrechtlich gerechtfertigt werden. Das ist häufig auch im Kontext von Menschenoder Grundrechten zu beobachten. Religiöse Ethik-Konzeptionen zählen ebenfalls zu den naturalistischen Konzeptionen, beziehen sich dann aber auf göttliche Gebote, wie z.B. das Gebot zum Zakat (Almosengeben) im Islam (vgl. Imran 2006). Auch wirtschaftspolitische Konzepte können eine (eher implizite) Ethik besitzen. So z.B. die Soziale Marktwirtschaft nach Alfred Müller-Armack, in der sich ein Recht auf Subsistenz aus der sozialen Gerechtigkeit (neben der Freiheit, das zweite große sittliche Ziel der Sozialen Marktwirtschaft) sowie den konkreten politischen Empfehlungen (wie sozialer Wohnungsbau, Mindestlöhne, Kinder- und Wohngeld etc.) ableiten lässt (vgl. Müller-Armack 1948). Damit deutet sich an, dass viele Ethik-Konzepte zwar durchaus Subsistenzaspekte enthalten, sie aber eben ein konkretes Subsistenzrecht - wie es z.B. von Henry Shue (1996) oder James C. Scott (1976) thematisiert wird - nur implizieren und es dann üblicherweise erst noch rekonstruiert werden muss. ${ }^{14}$

Im Gegensatz zu den erwähnten Konzepten soll die nachfolgende Subsistenzethik ganz bewusst die Gedanken zur Viabilität, Subsistenz und Zumutbarkeit ins Zentrum der Aufmerksamkeit rücken und sich von dort aus an die diskursethische Argumentation der Integrativen Wirtschaftsethik anlehnen. Subsistenzethik bedeutet demnach, die eigenen Handlungsabsichten daraufhin zu reflektieren, ob und wie sie das Subsistenzstreben Dritter beeinträchtigen. Diese Absichten sollen somit unter einen ethinach ökonomischer Methode, das Konzept einer Kernmoral nach Michael Baurmann, die transzendentale Wirtschaftsethik von Georg Trautnitz, die Islamische Wirtschaftsethik, die ethischen Implikationen der Sozialen Marktwirtschaft, das Naturrecht sowie das kritische Naturrecht nach Höffe. Für Details siehe Thieme (2012: 312-421). 
schen Legitimationsvorbehalt gestellt werden. ${ }^{15}$ Entsprechend impliziert die Subsistenzethik ein Subsistenzrecht als (überpositives) Moralprinzip, das jedem Menschen zusteht und folgende drei Ansprüche umfasst:

1. den Anspruch auf Viabilitätserhaltung, d.h. dass Möglichkeiten zum unmittelbaren Erhalt der Existenz in einer Gesellschaft zu gewähren sind;

2. den Anspruch auf Selbsthilfe und Subsistenzerbaltung, d.h. dass es über die Viabilitätserhaltung hinaus möglich sein muss, sich (selbst) zu verändern und an eine sich verändernde Umwelt anpassen zu können; sowie

3. den Anspruch darauf, dass Handlungsabsichten durch einen einvernehmlichen Diskurs ethisch zu legitimieren sind.

Der Anspruch auf Viabilitätserhaltung (1.) ist insofern eine absolute Notwendigkeit sozialer Beziehungen, als ein Zuwiderhandeln bedeuten würde, dem Gegenüber die Existenz abzusprechen und damit im Grunde das Desinteresse an einer sozialen Beziehung artikuliert wird. ${ }^{16}$ Das gilt selbstverständlich auch für wirtschaftliche Beziehungen: Wäre nicht sichergestellt, dass sich das Gegenüber viabel halten kann, käme es zu gar keiner Handels- bzw. Tauschbeziehung. Ein soziales Arrangement, das die Viabilität gefährdet, wäre außerdem nicht zumutbar.

Während der Anspruch auf Viabilitätserhaltung das Fundament für eine soziale Beziehung legt, zielt der Anspruch auf Subsistenzerhaltung (2.) darauf ab, die Individuen als mündige Akteure in Erscheinung treten zu lassen, d.h. ihnen die Möglichkeit an die Hand zu geben, überhaupt selbständig eine Entscheidung zu treffen und nicht nur auf - gleichwohl viable - regelhafte Reaktionsmechanismen reduziert zu werden.

Der Anspruch auf eine ethische Legitimation (3.) bedeutet, dass das legitime Interesse Dritter, selbst subsistieren zu können, in die eigenen Handlungsabsichten mit einzubeziehen ist. Der öffentliche Diskurs soll verhindern, dass die Interessen der (womöglich) Betroffenen bewusst (z.B. paternalistisch) oder unbewusst fehlinterpretiert oder gar völlig ausgeblendet werden, sie also gar kein Gehör finden und ihnen stattdessen ein Lebensentwurf diktiert wird, der den eigenen Entwürfen widersprechen kann, und insofern kaum Ergebnis einer freiwilligen Wahl gewesen wäre. Aus diesem Anspruch auf ethische Legitimation folgt als eine Konsequenz, dass Handlungsabsichten, die nicht durch einen einvernehmlichen Diskurs ethisch legitimiert wurden, von den Be-

15 Das hier entwickelte Verständnis von ,Subsistenzethik“ weicht davon ab, was James C. Scott (1976: 2ff., 167-179) als Subsistenzethik definiert, wird aber den dort enthaltenen Aspekt des Subsistenzrechts beibehalten und spezifizieren.

16 Diese Argumentation folgt in Anlehnung an die Rechtfertigung des Diskurses innerhalb der Integrativen Wirtschaftsethik (vgl. Ulrich 2008: 81-99), die auf der ,wechselseitige[n] Anerkennung von Gesprächspartnern als mündige (münd-ige!) Personen“ beruht, „die willens und prinzipiell in der Lage sind, vernünftig miteinander zu reden“ (Ulrich 2008: 82). Dem geht die Idee der „unbedingten Anerkennungswürdigkeit als ,unantastbare Person“" (Ulrich 2008: 47) innerhalb des ideellen Rollentauschs (Perspektivwechsels) voraus: „Daraus ergibt sich ihre [die Personen bzw. Argumentierenden, Anm. d. Verf.] prinzipielle moralische Gleichberechtigung bezüglich sämtlicher Voraussetzungen der Wahrung ihrer humanen Würde und damit ihrer personalen Identität und Autonomie“ (Ulrich 2008: 48). Siehe dazu auch Ulrich (2000: 557). 
troffenen als ethisch ungerechtfertigt ignoriert werden können. Dazu bedarf es allerdings zweier wichtiger Klarstellungen:

1. Der Umstand, dass Gesetze, Handlungen, usw. aus ethischer Sicht nicht legitim sein müssen, entbindet nicht davon, die eigenen Handlungsabsichten prinzipiell subsistenzethisch zu rechtfertigen. Das bedeutet, dass die jeweiligen Gegenreaktionen auf ethisch nicht legitime Gesetze, Handlungen, usw. nicht automatisch auch ethisch legitim sind!

2. Es mag eingewendet werden, dass die Vorstellung einer perfekten Kommunikationsgesellschaft, die der Integrativen Wirtschaftsethik zugrunde liegt und in der ein offener Diskurs über Subsistenzeinschränkungen möglich ist, eine Utopie darstellt. In der erfahrbaren Lebenswirklichkeit wird unseren Handlungsabsichten dagegen immer ein gewisser Mangel an Diskurs anhaften, denn die Einbindung aller Perspektiven - also auch jene, die von unseren Handlungsabsichten betroffen sein mögen - stellt eine kaum zu bewältigende Aufgabe dar. Das wiederum hat die Existenz eines Graubereichs zur Folge, in dem ethisch nicht legitime Handlungen geduldet werden. Dieser Bereich ist aber nicht mit der Zumutbarkeit gleichzusetzen. D.h. aus einer Duldung folgt nicht, dass die entsprechenden Zustände als zumutbar empfunden werden, denn auch unzumutbare Zustände mögen bis zu einem gewissen Grad geduldet sein. Den von Unzumutbarkeiten betroffenen Menschen können in dem Fall schlicht die Möglichkeiten fehlen, etwas an ihrer Lage zu verändern, weshalb ihnen nichts anderes übrig bleibt, als die Zustände zu (er-)dulden.

Ergänzend sei dazu ein Blick auf die Studien von James C. Scott (1976) gestattet, der zu Subsistenz, Subsistenzethik und Revolten im ländlichen Bereich Südostasiens forschte. ${ }^{17}$ Scott (1976: 203) wies u.a. darauf hin, dass in dem Maß, in dem (noch) Möglichkeiten existieren, um (kurzfristige) Engpässe in der Versorgung mit notwendigen Subsistenzmitteln abzufedern, auch die Wahrscheinlichkeit von direkten und gewalttätigen Revolten gemindert sei. ${ }^{18}$ Das legt den Schluss nahe, dass selbst nicht zumutbare Subsistenzeinschränkungen soweit geduldet sein mögen, wie sie nicht die Viabilität der Betroffenen beeinträchtigen. Problematisch ist darin, dass dieser Bereich der Duldung sehr dehnbar sein kann, die Grenzen dieser Duldung nicht immer sichtbar sein müssen und diese Duldung (gerade deshalb) von außen als Akzeptanz (fehl-) interpretiert werden könnte.

17 Die Erkenntnisse von Scott (1976) bezogen sich zwar auf landwirtschaftlich geprägte Gesellschaften, die sich von modernen Industrie- und Dienstleistungsgesellschaften unterscheiden (Rechte, Technik, usw.). Der elementare und universelle Charakter der von ihm beschriebenen Subsistenzphänomene provoziert jedoch eine Übertragung seiner Erkenntnisse auf moderne Volkswirtschaften (zumindest tendenziell). Scotts Buch legt es darauf auch deutlich an, wenn z.B. das Moralprinzip des Subsistenzrechts mit der Idee des gerechten Preises und der Lohnfrage in Verbindung gebracht wird (vgl. Scott 1976: 177).

18 Scott schrieb diesbezüglich von ,short-run subsistence needs“ (1976: 204), was in dem Kontext des von ihm etwas breiter angelegten Subsistenzbegriffs etwas missverständlich wirkt, weil er damit offensichtlich Nahrungsmittel, Saatgut, usw., also Subsistenzmittel, die maßgeblich darauf abzielen, was im vorliegenden Beitrag als Viabilitätserhaltung beschrieben wurde, meinte. 
Damit relativiert diese Duldung zwar den simplen Automatismus von unzumutbaren Subsistenzeinschränkungen und Widerstand, der vielleicht mit den Staatstheorien von Thomas Hobbes und John Locke assoziiert sein mag $^{19}$, doch gleichzeitig bedeutet dies keineswegs die Relativierung des Konfliktpotenzials, das aus unzumutbaren Einschränkungen resultieren könnte: Das Potenzial für gesellschaftliche Konflikte ist weiterhin vorhanden, aber es ist nur schwer in Erfahrung zu bringen, wann die Grenzen der Duldung erreicht sind und in welcher Form sich die Konflikte dann entladen. Dies gilt umso mehr für Fälle, in denen gar kein Interesse am gesellschaftlichen Diskurs besteht, d.h. gar nicht erst versucht wird, in Erfahrung zu bringen, wo die möglichen Grenzen der Zumutbarkeit respektive Duldung verlaufen.

In diesem Kontext sei außerdem darauf hingewiesen, dass neben aktiven Formen des Widerstands (Revolten) auch passive Formen existieren, die sich z.B. in Leistungsverweigerung, Zeitschinderei, Diebstahl oder „workplace violence“ äußern. ${ }^{20}$ Das Aufkündigen gesellschaftlicher Verhältnisse, die als unzumutbar empfunden werden, muss also nicht mit einem Paukenschlag einhergehen. Es ist denkbar, dass dieser gesellschaftliche Bruch im Klima einer Duldung und über längere Zeit hinweg - von Formen passiven Widerstands begleitet - (für andere) fast unbemerkt vollzogen wird. Auch darin liegt die besondere Relevanz des Bereichs der Duldung: Es ist dort eine Desintegration aus den bestehenden gesellschaftlichen Verhältnissen angelegt, die einen späteren Diskurs erschweren kann.

Der Anspruch auf die ethische Legitimation von Handlungsabsichten (Anspruch 3) soll deshalb zwar ausdrücklich keiner simplen Umsturz- bzw. Unterdrückungstheorie das Wort reden, aber ebenso nachdrücklich darauf hinweisen, dass Handlungsabsichten unter einem ethischen Legitimationsvorbehalt stehen und deshalb (nur) geduldet sein können. Um ethische Legitimierbarkeit zu erreichen, muss mindestens die Viabilitätserhaltung der von den Handlungsabsichten betroffenen Individuen gewährleistet sein. Soll die Handlungsabsicht ethisch legitimiert werden, ist sie einem offenen Diskurs zuzuführen, d.h., dass die betroffenen Parteien oder zumindest entsprechende Interessensverbände einzubeziehen sind. Im Bewusstsein einer imperfekten Kommunikationsgesellschaft bedeutet dies ebenso, eine ständige Dialogbereitschaft zu signalisieren. Diese Aspekte werden direkt einsichtig, wenn noch einmal die Schwierigkeiten bei der Bestimmung eines soziokulturellen Existenzminimums in Erinnerung gerufen werden: Gerade weil die Menschen in ihrem Viabilitäts- und Subsistenzerhalt von dieser Definition unmittelbar betroffen sind, die entsprechenden Festlegungen aber immer Werthaltungen und Interpretationen unterliegen, wäre sowohl die Einbindung von entsprechenden Interessensverbänden (wie z.B. Sozialverbänden) notwendig, als auch die ständige Dialogbereitschaft, um auf Unterversorgungen bzw. Viabilitätsbedrohungen aufmerksam zu machen und diese Missstände zu korrigieren.

\footnotetext{
19 Siehe dazu auch den Hinweis der ILO zum Risiko sozialer Unruhen (vgl. Fußnote 1).

20 Unter „workplace violence“ werden i.d.R. physische und/oder psychische Angriffe auf Personen am Arbeitsplatz verstanden - sowohl von „außen“ (z.B. durch Kundinnen und Kunden) als auch unter Kolleginnen und Kollegen selbst. Zur Vertiefung siehe z.B. ILO (2009) und Initiative Neue Qualität der Arbeit (2008). Ergänzend ließe sich dazu sicher auch z.B. die Gewalt gegen „Dinge“ (am Arbeitsplatz) anführen.
} 
Es ist außerdem zu beachten, dass die Wirkungen einer Handlungsabsicht nicht immer absehbar und zurechenbar sind: Im Fachjargon wird diesbezüglich von nicht intendierten Folgen intendierter Handlungen gesprochen. In diesem Kontext ließe sich kritisch fragen, wie dann überhaupt eine Handlungsabsicht ethisch legitimierbar sein kann, wenn doch immer die Gefahr besteht, das Subsistenzstreben Dritter unbeabsichtigt und vielleicht unmerklich - einzuschränken. In Anlehnung an die Integrative Wirtschaftsethik (vgl. Ulrich 2008: 90-94; 2000: 565) lässt sich eine Antwort auf diese Frage darin finden, im Bewusstsein der Möglichkeit nicht intendierter Folgen eine Mitverantwortung für eben diese zu übernehmen. Weil die Einschränkungen des Subsistenzstrebens nicht absehbar sind, bedeutet dies letztlich, den Mitmenschen ein Subsistenzrecht zu gewähren, das ihnen trotz der möglichen nicht intendierten und/oder nicht zurechenbaren Einschränkungen ihrer Subsistenz ein Subsistieren garantiert. Wer also ein Interesse an ethisch legitimierbaren Handlungen besitzt, muss bereit sein, den Mitmenschen ein Subsistenzrecht zu billigen und ein darüber hinaus reichendes (zumutbares) $\mathrm{Ma}$ an (Mit-)Verantwortung für die nicht intendierten Folgen der eigenen Handlungen zu übernehmen: Das Subsistenzrecht erweist sich auch vor dem Hintergrund dieser Mitverantwortung als eine Notwendigkeit zwischenmenschlicher Beziehungen.

\section{Die sozialstaatlichen Transfers aus subsistenzethischer Perspektive}

Die Ausführungen zu den ökonomischen Gründen, aus denen sich ein Subsistenzrecht rechtfertigen kann, deuten bereits an, welche Konsequenzen sich aus der Idee eines Subsistenzrechts ableiten lassen. Die im sozialpolitischen Kontext sicherlich wichtigsten Folgen sollen nachfolgend skizziert werden.

Dazu zählt in erster Linie die Notwendigkeit, den Gesellschaftsmitgliedern das Subsistieren zu gewährleisten. ${ }^{21}$ Andernfalls wäre jede Gesellschaftsform ethisch nicht zu rechtfertigen und allenfalls geduldet. Dort, wo diese Gewährleistung über Sozialtransfers umgesetzt wird, sollte beachtet werden, dass sich die damit verbundenen Ansprüche auf ein soziokulturelles Existenzminimum zumindest zum Teil bereits aus den Einschränkungen rechtfertigen, mit denen das Subsistieren jedes Individuums schon allein durch die Einbettung in den sozialen Kontext konfrontiert ist, und die auch als Vorleistungen interpretiert werden können. Zu denken wäre z.B. an:

- die Teilnahme am System der wettbewerblich organisierten Arbeitsteilung, was die absolute Abhängigkeit vom Erwerbslohn und das Risiko der Erwerbslosigkeit miteinschließt;

- die Bereitschaft zur beruflichen Qualifizierung und Spezialisierung, die hinsichtlich des Humankapitals mit der Bereitschaft zur Übernahme von Investitionsrisiken einhergeht;

- die Rechtschaffenheit im Sinne der Achtung einer existierenden Rechtsordnung und der Eigentumstitel im Speziellen. 
Diese Aspekte werden in der Debatte um die Ausgestaltung des Sozialstaates für gewöhnlich vernachlässigt. Eine Beachtung dieser Vorleistungen verträgt sich auch nicht mit einer sanktionsbewährten Workfare-Philosophie, wie sie in Deutschland unter der Chiffre Hartz praktiziert wird: Diese ist darauf ausgerichtet, den „Zustand eines Hilfebedürftigen möglichst unattraktiv“" (SVR 2007: 353) zu halten, weil sie davon ausgeht, dass sich die hilfsbedürftigen Menschen im Sozialtransfer einrichten könnten und dann keinen Anreiz besäßen, diesen Zustand wieder zu verlassen. ${ }^{22}$ Die Frage ist dabei, ob es den entsprechenden Personen in diesem Zustand überhaupt möglich ist, ihre Lage selbst zu verändern, also zu subsistieren. Wird ein Subsistenzrecht nicht allein schon durch das Ansinnen konterkariert, den Sozialtransfer unattraktiv zu halten?23

Deshalb ist es aus subsistenzethischer Sicht fragwürdig, die hilfsbedürftigen Menschen im Sozialsystem mit weiteren Einschränkungen ihres Subsistenzstrebens zu bedrohen, z.B. durch geringe Zumutbarkeitsschwellen zur Aufnahme von Tätigkeiten (Arbeitszwang) und Residenzpflichten. Praktisch werden dann die oben beschriebenen Vorleistungen ignoriert und den Bedürftigen paradoxerweise sogar zusät₹liche Einschränkungen auferlegt: Das Subsistieren hilfsbedürftiger Menschen, das in einer existenziellen Notsituation gewährleistet werden soll, wird auf diese Weise weiter eingeschränkt. Insofern liegt dort eine Missachtung des Anspruchs (2) auf Subsistieren vor. Dies trifft auch auf die Absicht zu, die Sozialtransfers „ungemütlich“ zu halten. Das kann dann aber auch soweit gehen, dass selbst der Anspruch (1) auf Viabilität verletzt wird: Das betrifft z.B. Sanktionen, die die Sozialtransfers - und somit das eigentlich zu garantierende Existenzminimum - kürzen.

$\mathrm{Zu}$ beachten ist außerdem die erwähnte Schwierigkeit, ein soziokulturelles Existenzminimum zu ermitteln. Aus subsistenzethischer Sicht wäre es dazu erforderlich, auch die Perspektive der Hilfsbedürftigen - zumindest in Vertretung durch Sozialverbände wie z.B. die Volkssolidarität, Erwerbslosenverbände, den Paritätischen Wohlfahrtsverband, usw. - einzubinden. Doch wie die Geschichte der Hartz-Reformen zeigt, wurde (und wird) diese Perspektive in stetiger Regelmäßigkeit gemieden. Das Ergebnis waren fachliche Mängel, vor allem in der Berechnung der Regelsätze, wie sie im Februar 2010 vom Bundesverfassungsgericht beanstandet wurden (vgl. BVerfG 2010). Selbst die davon ausgelöste Nivellierung der entsprechenden Gesetze, die rückwirkend zum 01.01.2011 in Kraft trat, scheint hinsichtlich der Ermittlung der Sozialtransfers erneut mit handwerklichen Mängeln behaftet zu sein (vgl. Becker 2011 und Münder 2011). Diese Mängel schlagen sich dann unmittelbar in der Beeinträchtigung des Anspruchs auf Viabilitäts- und Subsistenzerhaltung nieder. Ferner zeigt sich, wie elementar die Missachtung des Anspruchs (3) auf ethische Legimitierung auf genau diese

22 Dieser Logik folgen auch die deutschen und britischen Sparprogramme im Rahmen der wirtschaftlichen Krisenbewältigung: „Großbritannien und Deutschland beispielsweise sparen bei den Bedürftigsten und den Sozialversicherungsbeziehern mit dem Ziel, den Lohnabstand zu reduzieren und den Anreiz, eine Beschäftigung aufzunehmen, zu erhöhen“ (Heise und Lierse 2011: 224). Zum Begriff „Workfare“ siehe Lødemel (2005) und Dingeldey (2006). Zur kritischen Auseinandersetzung mit der Workfare-Philosophie siehe Spindler (2008), Wolf (2008), Wölfle und Schöller (2004) sowie Zilian (2000).

23 Siehe dazu auch Wacquant (2009: 69ff.), der beschreibt, wie die Bürokratie des amerikanischen Sozialsystems die Bedürftigen davon abhalten kann, ihre Ansprüche geltend zu machen. 
Ansprüche wirkt: Ohne einen offenen Diskurs kann gar nicht in Erfahrung gebracht werden, ob die Viabilität der EmpfängerInnen von Sozialtransfers überhaupt gewährleistet ist und welche Einschränkungen des Subsistierens zumutbar erscheinen.

Insgesamt überwiegt damit der Eindruck, dass die Ermittlung des Existenzminimums als eine rein technische Angelegenheit behandelt wurde (und wird), bei der die Frage, ob dieses Existenzminimum dann tatsächlich angemessen und zumutbar ist, und die ethische Legitimierbarkeit nur eine untergeordnete Rolle spielten. Dieser Missstand ließe sich zukünftig vermeiden, wenn tatsächlich ein Interesse an einem redlichen Diskurs bestünde, der u.a. die Perspektive der Betroffenen miteinbezieht.

Vor dem Hintergrund, dass die Sozialtransfers des Staates bisweilen mit dem Mindestlohn gleichgesetzt werden, bedarf es dazu noch einer kurzen Klarstellung. ${ }^{24}$ Wichtig ist, dass sich der Mindestlohn auf ein Arbeitsverbältnis bezieht und er sich damit deutlich vom bloßen Sozialtransfer (ohne Arbeit) unterscheidet. Der Mindestlohn muss deshalb nicht nur die Viabilität und ein gesellschaftlich zu verhandelndes Maß an Subsistenz ermöglichen, sondern hat darüber binaus jene Einschränkungen zu einem Mindestmaß zu kompensieren, die ganz konkret mit dem Arbeitsverhältnis einhergehen, aufgrund der Marktmachtverhältnisse aber nicht in einer freien Lohnverhandlung eingefordert werden können (z.B. Wegzeiten, Flexibilität und Mobilität). Deshalb ist es falsch, die (reinen) Sozialtransfers des Sozialstaates als Mindestlohn zu deklarieren. Aus ähnlichen Gründen ist es falsch, die Sozialtransfers mit dem Argument senken zu wollen, dass damit der Lohnabstand gewahrt bleibe. Dies würde gegen das Moralprinzip eines Subsistenzrechts verstoßen, weil es die Ansprüche auf Subsistenzmittel (z.B. in Form eines soziokulturellen Existenzminimums) ignoriert. Zum Anderen wären damit ebenfalls die berechtigten Ansprüche auf Kompensation der mit dem Arbeitsverhältnis einhergehenden Einschränkungen ignoriert: Der durch gesenkte Sozialtransfers erzeugte Lohnabstand kaschiert diesen Missstand nur. ${ }^{25}$

\section{Abschließende Gedanken}

Der im vorliegenden Beitrag skizzierten Subsistenzproblematik mag der Vorwurf gemacht werden, dass sie doch nur eine Banalität beschreibe. Wer das erwähnte Argument in Erinnerung ruft, dass die (Selbst-)Erhaltung der Arbeitskräfte schlicht notwendig sei, um einen wirtschaftlichen Prozess fortzusetzen, der oder die mag dieser Kritik zustimmen. Doch trotz dieser Banalität ist es sehr erstaunlich, wie gering die Ökonomen die Bedeutung der Subsistenz einzuschätzen scheinen. Das lässt sich daran ablesen, dass die wesentlichen Arbeiten zur Subsistenz - nämlich die von Henry Shue (1996) und James C. Scott (1976) - außerhalb der Ökonomik geschrieben wurden, obwohl sie deutlich in einem ökonomischen Kontext standen. ${ }^{26}$ Diese Erkennt-

Diese Klarstellung erhebt freilich nicht den Anspruch, den Mindestlohn in diesem Text erschöpflich diskutieren zu wollen.

25 Die Konsequenzen eines Subsistenzrechts als Moralprinzip beschränken sich nicht nur auf die Frage der Löhne und Sozialtransfers, sondern sie erstrecken sich auch auf die Frage des Eigentums in der Gesellschaft (Welche Eigentumskonzentration ist zumutbar?), die Modellierung des Arbeitsmarktes in der Ökonomik, auf den Zugang zu Wohnraum, die Problematik von (illegalen) Landbesetzungen, usw. Das lässt sich im Rahmen dieses Beitrags aber nicht weiter vertiefen. Zum Beispiel setzte Shue (1996: 23) die Subsistenz mit „minimal economic security“ gleich. 
nisse drangen bisher nicht in die Ökonomik vor. Den Studierenden der Ökonomik wird im Studium zwar viel über die Kalkulation von optimalen Lohnhöhen und Arbeitsmarktgleichgewichten vermittelt, aber ohne dabei die Fundamentalfunktion von Löhnen - die Subsistenzerhaltung - einzubeziehen.

Für Ökonominnen und Ökonomen erwächst daraus ein nicht unerhebliches Problem, denn, wenn sie bei ihren Empfehlungen zum Arbeitsmarkt und zur Sozialpolitik die Subsistenzfragen übergehen, sind subsistenzethische Konflikte praktisch vorprogrammiert - über einen entsprechenden Mangel an Akzeptanz dürfen sie sich dann nicht wundern. Die eigenen ökonomischen Ansätze und Empfehlungen an der Subsistenzethik zu prüfen, das könnte dagegen die Akzeptanz der ökonomischen Empfehlungen erhöhen. Gleichzeitig wäre die Ökonomik damit auch wieder näher an ihre ursprüngliche Aufgabe gerückt - dem „guten Leben“ zu dienen.

\section{Literaturverzeichnis}

Becker, I. (2011): Bewertung der Neuregelungen des SGB II, in: Soziale Sicherheit, Sonderheft, $7-62$.

Berlin, I. (1995): Freiheit. Vier Versuche, Frankfurt a.M.: S. Fischer.

BV erfG (2010): Leitsätze zum Urteil des Ersten Senats vom 9. Februar 2010. BvL 1/09, Link: http://www.bverfg.de/entscheidungen/ 1s20100209_1bvl000109.html (zuletzt abgerufen am: 30.04.2013).

Chalmers, A. F. (2007): Wege der Wissenschaft, sechste Auflage, Berlin, Heidelberg, New York: Springer.

Dingeldey, I. (2006): Aktivierender Wohlfahrtsstaat und sozialpolitische Steuerung, in: Aus Politik und Zeitgeschichte, Jg. 2006/Heft 8-9, 3-9.

Habermas, J. (1958): Philosophische Anthropologie (ein Lexikonartikel), in: Habermas, J. (Hrsg.) (1973): Kultur und Kritik. Verstreute Aufsätze, Frankfurt a. M.: Suhrkamp, 89117.

Hauser, R. (2008): Das Maß der Armut, in: Huster, E.-U./Boeckh, J./Mogge-Grotjahn, H. (Hrsg.): Handbuch Armut und Soziale Ausgrenzung, Wiesbaden: VS Verlag für Sozialwissenschaften, 94-117.

Heise, A./Lierse, H. (2011): Wirtschaftskrise, Austeritätspolitik und das Europäische Sozialmodell, in: Wirtschaft und Gesellschaft, Jg. 37/Heft 2, 201-230.

ILO (2013): ILO-Bericht zur „Welt der Arbeit“, Link: http://www.ilo.org/berlin/ presseinformationen/WCMS_209978/lang--de/index.htm (zuletzt abgerufen am: 28.04.2013).

ILO (2009): Violence at Work - A major Workplace Problem, Link: http://www.ilo.org/wcmsp5 /groups/public/---ed_protect/---protrav/---safework/documents/genericdocument/ wcms_108531.pdf (zuletzt abgerufen am: 01.05.2013).

Imran, H. (2006): Das islamische Wirtschaftsrecht, Bremen: Salzwasser Verlag.

Initiative Neue Qualität der Arbeit (2008): Gewaltfreier Arbeitsplatz, Bundesanstalt für Arbeitsschutz und Arbeitsmedizin (Hrsg.), Dortmund, Link: http://www.inqa.de/Shared Docs/PDFs/DE/Publikationen/gewaltfreier-arbeitsplatz.pdf (zuletzt abgerufen am: 05.05.2014).

Lodemel, I. (2005): Workfare, in: CESifo DICE Report, No. 2, 13-17.

Müller-Armack, A. (1981/1948): Vorschläge zur Verwirklichung der Sozialen Marktwirtschaft, in: Dürr, E. (Hrsg.): Genealogie der Sozialen Marktwirtschaft, Ausgewählte Werke von Alfred Müller-Armack, zweite Auflage, Bern: Haupt, 90-109. 
Münder, J. (2011): Verfassungsrechtliche Bewertung, in: Soziale Sicherheit, Sonderheft, 63-94.

Scott, J. C. (1976): The Moral Economy of the Peasant, New Haven, London: Yale University Press.

Shue, H. (1996): Basic Rights, Princeton und New Jersey: Princeton University Press.

Spindler, H. (2008): Arbeiten für die Grundsicherung, in: Soziale Sicherheit, Zeitschrift für Arbeit und Soziales, Jg. 57/Nr. 11, 365-372.

SVR (2007): Das Erreichte nicht verspielen, Wiesbaden, Link: http://www.sachverstaendigenratwirtschaft.de/fileadmin/dateiablage/download/ gutachten/jg07_ges.pdf (zuletzt abgerufen am: 09.11.2012).

Thielemann, U. (2010): System Error. Bonn: Bundeszentrale für politische Bildung.

Thieme, S. (2010): Subsistenz: Geschichte, Bedeutung und Rekonstruktion des Subsistenzbegriffes, in: MPRA Paper, Nr. 24553, Link: http://mpra.ub.uni-muenchen.de/24553/ (zuletzt abgerufen am: 28.04.2013).

Thieme, S. (2012): Das Subsistenzrecht: Begriff, ökonomische Traditionen und Konsequenzen, Dissertation, Marburg.

Trautnitz, G. (2008): Normative Grundlagen der Wirtschaftsethik: Ein Beitrag zur Bestimmung des Ausgangsparadigmas, Berlin: Duncker \& Humblot.

Ulrich, P. (2000): Integrative Wirtschaftsethik: Grundlagenreflexion der ökonomischen Vernunft, in: Ethik und Sozialwissenschaften, Vol. 11/No. 4, 555-567.

Ulrich, P. (2008): Integrative Wirtschaftsethik, vierte Auflage, Bern, Stuttgart, Wien: Haupt.

Wacquant, L. (2009): Bestrafen der Armen, Opladen, Farmington Hills: Barbara Budrich.

Wölfle, T./Schöller, O. (2004): Soziale Disziplinierung im flexiblen Kapitalismus: Die kommunale „Hilfe zur Arbeit“, in: PROKLA, Jg. 34/Heft 136, 339-356.

Wolf, M. (2008): Hartz IV-Sofortangebote als Workfare Element, in: Soziale Sicherheit, Zeitschrift für Arbeit und Soziales, Jg. 57/Heft 11, 372-379.

Zilian, H. G. (2000): Aktivierung und Workfare, Arbeitsmarktpolitische Herrschaftsinstrumente in der flexibilisierten Wirtschaft, in: PROKLA, Jg. 30/Heft 121, 567-584. 